\title{
¿Sirven las políticas públicas restrictivas al consumo de alcohol y de manejo responsable para disminuir las muertes por choques de vehículos?
}

\author{
Are restrictive public policies related to alcohol consumption effective for the reduction of motor vehicle crash fatalities?
}

\section{Comentado de:}

Naimi et al. JAMA Intern Med. 2018:178(7) 894-9011

\section{Objetivos}

Examinar la asociación entre las políticas restrictivas respecto del consumo de alcohol y la probabilidad de que las muertes por traumas viales estén vinculadas con esta sustancia en los Estados Unidos (EE.UU.)

\section{Diseño}

Estudio observacional de datos agregados.

\section{Medición de resultados principales}

Probabilidad de que ante una muerte por un choque de un vehículo motorizado se documente en al menos uno de los conductores una concentración de alcohol en sangre mayor a 0,08\%.

\section{Evaluación de factores de riesgo}

Para todos los análisis que involucran a personas con muertes consideradas por consumo de alcohol, se agruparon los resultados en 10 conglomerados de imputación para calcular la estimación central y el error estándar. Se utilizaron modelos de regresión logística para evaluar la asociación entre las políticas y las proba- bilidades de que una fatalidad por choque estuviera relacionada con el alcohol. Debido a que hay un retraso entre la promulgación y la implementación de la política, se utilizó un retraso de un año para analizar el datos. En los análisis ajustados, las covariables a nivel individual incluían la edad, el sexo, la raza/etnia y el año. Las covariables a nivel estatal incluyeron la proporción de hombres, las proporciones de raza/etnia, la proporción de la población de al menos 21 años, el nivel de urbanización, el ingreso medio del hogar, la proporción de individuos con educación universitaria, las tasas de vigilancia del estado y el número medio de millas recorridas por vehículo por persona.

\section{Resultados}

Desde 2000 a 2015 se documentaron en los EE.UU. 505.614 muertes por choques de vehículos motorizados, 35,4\% de ellas relacionadas con el consumo de alcohol. Cada diez puntos porcentuales de incremento en el puntaje que valora el grado de restricción de la política estatal respecto del consumo de alcohol (Alcohol Policy Scale, APS) se observó una disminución de aproximadamente $10 \%$ en la probabilidad a nivel individual de el alcohol hubiera participado en un trauma vial mortal (ver Tabla 1).

Tabla 1. Razón de probabilidad ajustada de que una fatalidad de accidente automovilístico en un adulto estuviera relacionada con el alcohol asociada con un aumento de 10 puntos porcentuales en el puntaje A/cohol Policy Scale en los Estados Unidos.

\begin{tabular}{|c|c|c|c|c|}
\hline & $\begin{array}{c}\text { Todas las muertes } \\
\text { OR (IC95\%) }\end{array}$ & $\begin{array}{c}\text { Conductores } \\
\text { OR (IC95\%) }\end{array}$ & $\begin{array}{c}\text { Pasajeros } \\
\text { OR (IC95\%) }\end{array}$ & $\begin{array}{c}\text { Otros } \\
\text { OR (IC95\%) }\end{array}$ \\
\hline Total de la muestra & $0,90(0,89$ a 0,91) & $0,91(0,90$ a 0,92) & $0,89(0,87$ a 0,92$)$ & $0,91(0,88$ a 0,94$)$ \\
\hline
\end{tabular}

OR: Odds Ratio; IC 95: Intervalo de confianza del $95 \%$.

Fuente: Sistema de informes de análisis de fatalidad de los Estados Unidos.

\section{Conclusiones}

El fortalecimiento de las políticas sobre el alcohol, incluidas las que no se dirigen específicamente a las restricciones en la conducción de automóviles, podría reducir las muertes por accidentes relacionados con el alcohol. Las políticas también pueden proteger contra accidentes fatales que involucran niveles de alcoholemia por debajo del límite legal actual para conducir en los EE.UU.

Conflicto de interés de los autores: Ninguno

Fuente de financiamiento: Insituto Nacional de Salud (EEUU)

\section{Comentario}

Los choques de vehículos motorizados lideran las causas de mortalidad. Sin embargo la asociación entre la restrictividad de los entornos por políticas de alcohol y las muertes relacionadas al consumo de alcohol no fueron relacionadas previamente. A medida que se fortalecen las políticas restrictivas sobre el alcohol, incluyendo las restricciones al consumo y las restricciones al manejo de vehículos motorizados, disminuyen las muertes por choques relacionados con el alcohol.

Los autores valoraron las políticas sobre alcohol en cada estado utilizando la APS ${ }^{2,3}$, y las correlacionaron con la posibilidad de morir en un accidente relacionado con el alcohol. Cuanto más restrictivo era un entorno para el alcohol, menor era la posibilidad de morir a causa de un choque de vehículo motorizado en el que el alcohol estuviera involucrado.

Si bien la correlación no es lineal, por aproximadamente cada 10\% mayor el valor del APS (y por lo tanto $10 \%$ más restrictivos) disminuirían $10 \%$ las muertes por vehículos motorizados en los cuales por lo menos un conductor había bebido alcohol. 
Es importante destacar que en este estudio se consideró que una muerte estaba relacionada con el alcohol a aquella en la que al menos uno de los conductores tenía un nivel de esa sustancia en sangre mayor al $0,08 \%$.

Sería interesante conocer en Argentina, con las diferencias que se detectan en los límites legales de alcoholemia para conducir entre provincias y diferentes políticas públicas restrictivas hacia el alcohol, cuál es la eficacia en prevenir la mortalidad relacionada con los accidentes vehiculares, información con la que por el momento, nos estamos debiendo.

\section{Conclusiones del comentador}

Las políticas públicas restrictivas sobre el alcohol, incluidas las que no se dirigen específicamente a la conducción de automóviles bajo el efecto de esta sustancia, podrían ayudar a reducir las muertes ocasionadas por colisiones vehiculares.

Lucas Esteban Figueroa [ Servicio de Medicina Familiar y Comunitaria, Hospital Italiano de Buenos Aires, Universidad Católica Argentina lucas. figueroa@hospitalitaliano.org.ar ]

Figueroa LE ¿Sirven las políticas públicas restrictivas al consumo de alcohol y de manejo responsable para disminuir las muertes por choques de vehículos?. Evid Actual Pract Ambul. 2019;22(2):e001110. Comentado de: Naimi TS, Xuan Z, Sarda V, Hadland SE, Lira MC, Swahn MH, Voas RB, Heeren TC. Association of State Alcohol Policies With Alcohol-Related Motor Vehicle Crash Fatalities Among US Adults. JAMA Intern Med. 2018 Jul 1;178(7):894-901.

\section{Referencias}

1. Naimi TS, Xuan Z, Sarda V, Hadland SE, Lira MC, Swahn MH, et al. Association of State Alcohol Policies With Alcohol-Related Motor Vehicle Crash Fatalities Among US Adults. JAMA internal medicine. 2018;178(7):894-901. Available from: 10.1001/jamainternmed.2018.1406.

2. Naimi TS, Blanchette J, Nelson TF, Nguyen T, Oussayef N, Heeren TC, et al. A new scale of the U.S. alcohol policy environment and its relationship to binge drinking. American Journal of Preventive Medicine. 2014;Available from: 10.1016/j.amepre.2013.07.015.

3. Bloss G. The Alcohol Policy Information System (APIS) and policy research At NIAAA. Alcohol research health: the journal of the National Institute on Alcohol Abuse and Alcoholism. 2011;34(2):246-7. 